\title{
A New Technological Platform for the Multisite Assessment of 3D Seismocardiogram and Pulse Transit Time in Cardiac Patients
}

\author{
Marco Di Rienzo, Prospero Lombardi, Diana Scurati, Emanuele Vaini
}

Fondazione Don Carlo Gnocchi, Dept of Biomedical Technology, Milano, Italy

\begin{abstract}
In this paper we present a new hardware architecture, the SeisMote system, allowing a multisite assessment of seismocardiogram (SCG), photoplethysmogram (PPG) and pulse transit time (PTT). The whole architecture is based on a number of miniaturized electronic nodes, designed to accommodate different types of sensors, and a small-sized hub.

In the present implementation the system may handle 5 nodes, and each node includes a SCG and a PPG sensor, from which PTT may also be derived. The hub collects data from the nodes and provides the additional measure of one ECG channel. Data are locally stored and transmitted via Bluetooth to external devices for online alerts and analyses. Currently, recordings on cardiac patients are in progress to investigate the feasibility of a clinical use of the system, in view of its possible integration in telemedicine services.
\end{abstract}

\section{Introduction}

In recent years there has been a growing interest in the seismocardiogram (SCG), photoplethysmogram (PPG) and pulse transit time (PTT) signals because of their rich biological content, simplicity in their assessment and integrability in telemedicine and teleriabilitation services.

At this moment, SCG and PPG are usually collected by using separate devices with each signal detected by a single sensor.

In this paper we present a new hardware architecture, the SeisMote system, that enhances the above measuring approach and allows a simultaneous multisite assessment of SCG, PPG and PTT. The system has been developed to guarantee flexibility of use. Among the many possible configurations this architecture provides us with a simultaneous SCG evaluation from multiple precordial locations, and PPG and PTT estimates from different vascular sites.

Details of the system and preliminary examples of application are described in the following sections.

\section{The measured signals}

In this section, we briefly describe the signals recorded by the SeisMote system.

- SCG is the measure of the precordial vibrations produced by the beating heart and the blood ejection from the ventricles into the vascular tree. This signal may be detected by placing an accelerometer on the chest surface, usually on the lower part of the sternum. SCG provides information on different mechanical events of the cardiac cycle, including opening and closure of the aortic and mitral valves, atrial systole, isovolumic contraction and relaxation phases. The typical SCG waveform is illustrated in the mid panel of fig. 1 . Details on this signal and its derived parameters may be found in [1].

- PPG is the measure of the light absorbed by the blood flowing into the vessels. The PPG sensor is typically composed of a light source (commonly a LED) and one or more detectors able to collect the light passing-through or being back-scattered by the blood. An example of PPG waveform is shown in the lower panel of figure1. Through this signal it is possible to estimate changes in the vessel diameter over time and thus estimate the arrival of the blood pressure pulse in a given location of the vascular tree [2]. The same methodology is commonly used for estimating the blood oxygen saturation, by using red and infra-red LEDs.

- PTT is defined as the measure of the time needed by the blood pressure wave to travel from the aorta to a distal arterial site. PTT inversely depends on both the arterial stiffness and blood pressure. By definition the PTT should be estimated as the time delay between the aortic valve opening (AO) and the pulse arrival to a distal (finger tip, ear lobe, forehead) or proximal (sternum [3]) artery. While the pulse arrival may be easily detected by different technologies (PPG or tonometry), the AO detection is a complex task if achieved by traditional approaches. For this reason the R peak of the ECG, instead of the AO, is often used as starting point for the transit time measure. This new index is commonly termed Pulse Arrival Time (PAT) and provides a distorted estimate of PTT because it includes the spurious contribution of most of the time delay from the electrical 


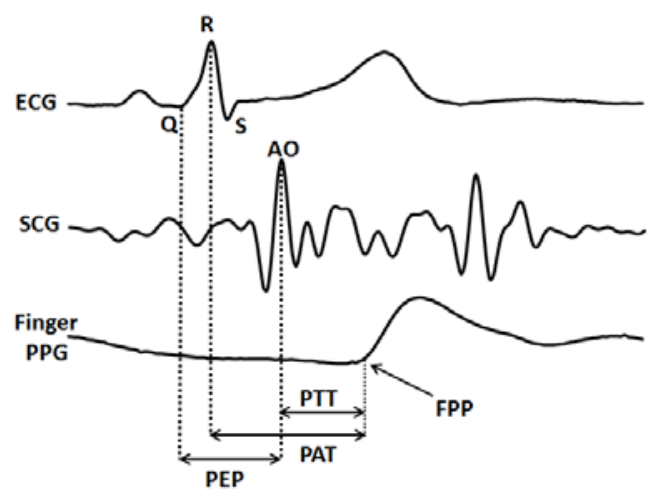

Figure 1 - Typical ECG, SCG and finger PPG waveforms and the fiducial points used for the estimation of PEP, PTT and PAT. AO is the SCG fiducial point associated with the opening of the aortic valve. FPP $=$ Foot of the Pulse Pressure wave. See text for the other abbreviations.

depolarization of the left ventricle to the opening of the aortic valve (the pre-ejection period, PEP).

Recently, we proposed the use of SCG not only to get information on cardiac mechanics, but also as a mean to identify AO in the PTT estimation [4]. A schematization of this approach is illustrated in fig. 1.

\section{The hardware architecture}

The SeisMote system is composed of a number of miniaturized multisensor nodes, and a hub (see figure 2). In the current first implementation the architecture may accept up to 5 nodes.

\subsection{The sensorized nodes}

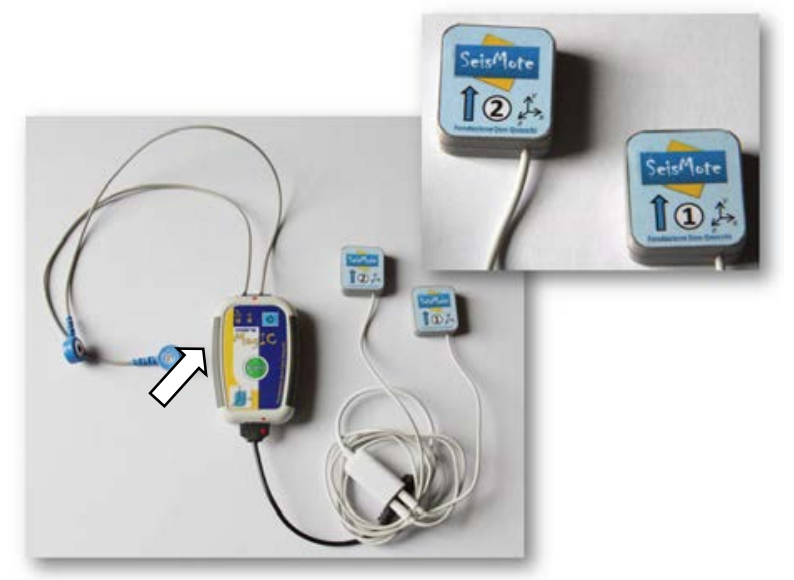

Figure 2 - The wired version of the SeisMote system including the hub (indicated by the arrow) with the connections for the ECG electrodes and the sensor nodes. Inset: detail of the sensor nodes.
Each node has the size of a coin, includes four 3d MEMS accelerometer for the SCG measure (Freescale MMA8451Q), and a red/infrared photopletysmograph (Maxim Max30100). All signals are sampled at $200 \mathrm{~Hz}$. At each $A / D$ conversion the samples from the four accelerometers are averaged so to halve the SCG noise. Thus, the node provides five signals: 3 low-noise acceleration axes and two PPG channels (red and infrared). Signals may be individually selected and transmitted to the hub via cable or via an RF wireless connection. At this moment only the wired version of the nodes is available and the wireless type is under development. The nodes may be positioned in different body locations by adhesive tape, velcro strips or clips. The wired implementation of the nodes is shown in the inset of figure 2. Although the present platform is focused on SCG, PPG and PTT, the nodes have been designed so to easily accommodate a large variety of additional sensors, including gyroscopes, thermometers, respiratory sensors, skin resistance detectors.

\subsection{The hub}

The electronic module that receives data from the nodes has the size of $50 \times 70 \mathrm{~mm}$ and weight of $70 \mathrm{~g}$. This module is an enhancement of the electronics used to collect physiological signals from a sensorized vest during sleep aboard the International Space Station in 2015 [5]. This module collects data from the sensorized nodes and allows the measure of one ECG lead by standard adhesive AgCl electrodes. All signals may be locally stored and transmitted in real time to a remote device (pc, tablet or smartphone) via Bluetooth.
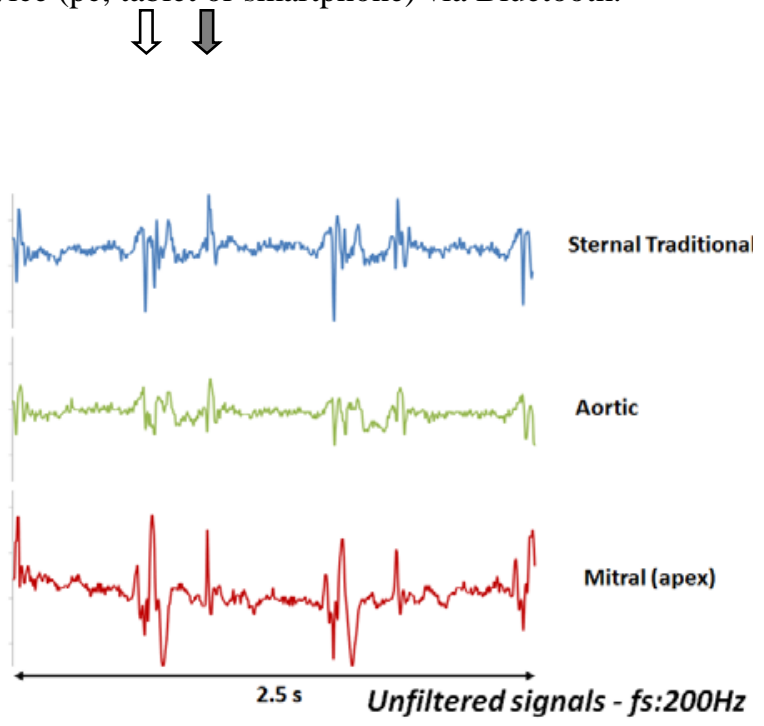

Figure 3 - Dorso-ventral SCG components detected by three nodes of the system placed on different chest locations (see text for details) in a healthy subject. The white and gray arrows indicate the start of the systolic and diastolic part of the heart cycle, respectively. 


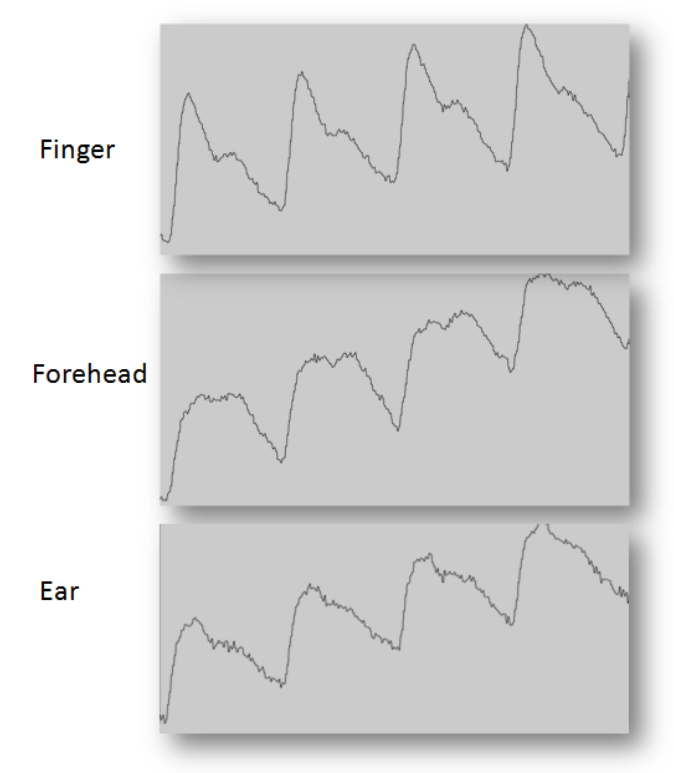

Figure 4 - Example of PPG measured at different vascular sites.

\section{Examples of collected data}

As indicated in the Introduction, the system is designed to guarantee flexibility of use. Among the many possible configurations this architecture allows us: 1) to simultaneously evaluate SCG from different precordial locations (by placing several nodes on the chest), 2) to obtain multiple PPG and PTT estimates on different vascular sites, and 3) to estimate PTT by a single node placed on the sternum (because of the concomitant presence of the SCG and PPG sensors in the same node).

The potentialities of the system are shown by the following examples.

Figure 3 illustrates a simultaneous SCG multisite recording obtained by using three sensor nodes. Only the dorso-ventral acceleration components of the SCG are shown in the figure. Nodes were placed on the lower part of the sternum, on the chest in correspondence with the 2nd right intercostal space (the position for the aortic valve auscultation) and on the heart apex (the position for the mitral valve auscultation). It is apparent that although the start of the systolic and diastolic phases of the cardiac cycle are obviously synchronized among the three signals (see arrows), the waveform profiles are different. Actually, each signal puts in particular evidence specific aspects of cardiac mechanics according to the sensor position on the chest. Thus, from this first observation it appears that the multisite SCG assessment may actually provide a more informative picture of cardiac mechanical events than a single site measure.

Figure 4 shows three PPGs measured on three different artery sites: finger tip, forehead and ear. This approach might facilitate the comparison of control strategies in different hemodynamical districts through the analysis of

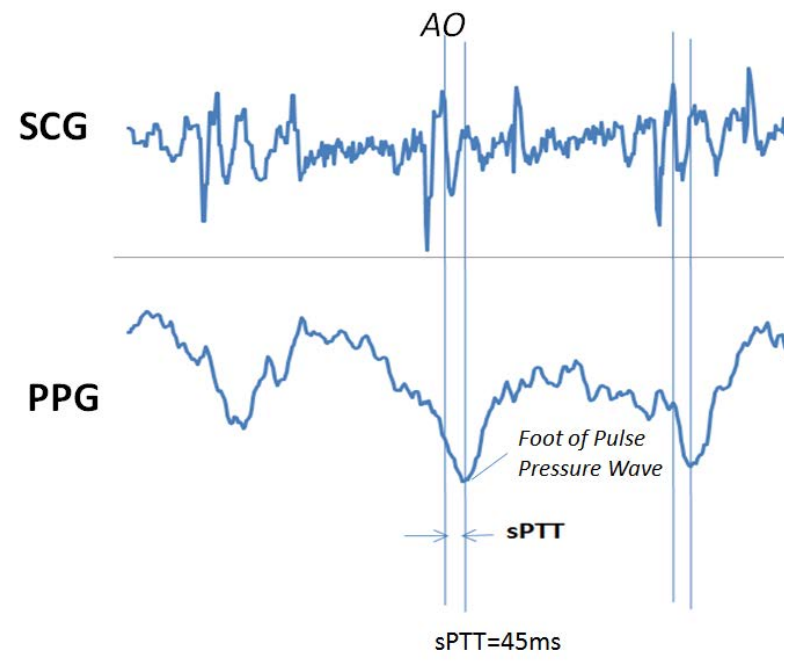

Figure 5 - SCG and PPG detected by a single node placed on the sternum in a healthy subject, and the derived sternal PTT (sPTT).

the different morphological PPG waveforms, and, if the SCG is also recorded, by simultaneous PTT estimates obtained at different arterial sites.

Finally, figure 5 illustrates an example of the SCG and PPG signals measured on the sternum by a single node with the derived sternal PTT.

\section{Toward clinical applications}

The SeisMote system has been developed to be integrated in a telemedicine service for the remote monitoring of discharged cardiac patients and as a tool for the evaluation of improvements in the cardiac mechanical performance of post-event patients during their physical rehabilitation programs.

Recently, we used the system to investigate if the SCG sensor placement on different parts of the sternum might influence the SCG waveform.

Eight patients suffering from cardiac heart failure were recruited for this study. Each subject remained in supine position for 5 minute during which three SCG signals were simultaneously recorded by positioning three sensor nodes of our device on the upper (u), mid (m) and lower (l) part of the sternum. We denoted the output signals as SCGu, SCGm and SCGl, respectively. From each recording three ensemble waveforms of the dorso-ventral components of the SCG signals were estimated by using the ECG R peak for synchronization. An example of the 3 ensemble SCG waveforms obtained in a patient are shown in figure 6. Individual differences in the SCG profiles were quantified by estimating the zero lag crosscorrelation coefficient (CC) between SCGu and SCGm, SCGu and SCGl, SCGm and SCGl. A CC value $<0.8$ was taken as indicative of a marked shape 


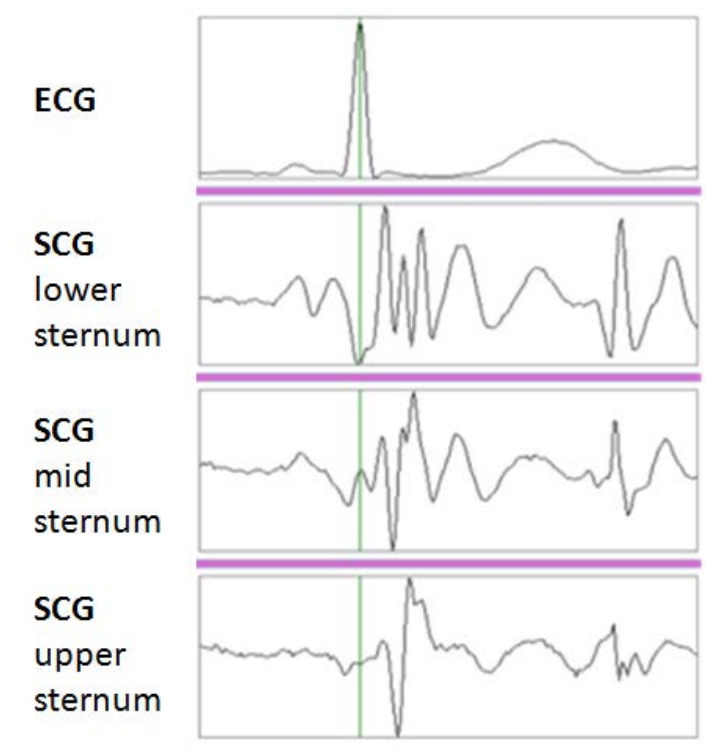

Figure 6 - Example of simultaneous multisite SCG assessment on the sternal area of a patient suffering from diastolic heart failure.

discrepancy.

Important differences were found between the SCG waveforms detected in the upper and lower part of the sternum (average $\mathrm{CC}=0.44$ ), while in most subjects SCGm and SCGl profiles are pretty similar (average CC= 0.88). Magnitude and characteristics of the SCG discrepancies were largely individual and no general pattern could be found. Interestingly, we also observed that in several patients the best sensor position to obtain a waveform approaching the reference SCG profile proposed by Crow et al. [1] was the upper sternum, and not the lower sternum, as commonly selected.

These preliminary results foster further investigations on the most appropriate location of the sensor for the SCG assessment in cardiac patients.

\section{Conclusions}

In this paper a new system for the multisite assessment of SCG, PPG and PTT has been presented. From the methodological perspective, the data collection we are carrying out on patients by this device provides evidence of the feasibility of the SCG measure and of the ease of use of the system. These findings represent a positive support to next activities aimed at the inclusion of the device into telemedicine platforms for the remote monitoring of cardiac patients. In addition, with the wireless version of the nodes, expected for the second part of 2016, m-Health applications will be also developed for providing the patients with a real time feedback on their cardiac mechanics in daily life through the smartphone.

\section{References}

[1] Inan O, Migeotte PF, Park KS, Etemadi M, Tavakolian K, Casanella R, Zanetti J, Tank J, Funtova I, Prisk GK, Di Rienzo M. Ballistocardiography and Seismocardiography: A Review of Recent Advances. J Biomed Health Inform 2015 Jul;19(4):1414-270.

[2] Tamura T, Maeda Y, Sekine M, Yoshida M. Wearable Photoplethysmographic Sensors-Past and Present. Electronics 2014, 3, 282-302; doi:10.3390.

[3] Sola J, Chetelat O, Sartori C, Allemann Y, Rimoldi SF. Chest Pulse-Wave Velocity: A Novel Approach to Assess Arterial Stiffness. IEEE Trans Biomed Eng, vol. 58, no. 1, Jan 2011: 215-223

[4] Di Rienzo M, Vaini E, Lombardi P. Use of seismocardiogram for the beat-to-beat assessment of the Pulse Transit Time: A pilot study. In: Proc IEEE Eng Med Biol Soc. 2015 Aug;2015:7184-7.

[5] Di Rienzo M, Vaini E, Lombardi P. Wearable Monitoring: a Project for the Unobtrusive Investigation of Sleep Physiology Aboard the International Space Station. Computing in Cardiology 2015;42.

Address for correspondence:

Marco Di Rienzo

Fondazione Don Carlo Gnocchi ONLUS

Dept. of Biomedical Technology

Via Capecelatro 66, 20148 Milano, Italy

E-mail address: mdirienzo@dongnocchi.it 